\title{
Consumers' Awareness and Acceptability towards Online Grocery Shopping: A Study on the Consumers of Dhaka Metropolitan City
}

\author{
Hamidul Islam $^{1}$, Stanley Sumon Rodrick ${ }^{2}$, Md. Abu Jubaer ${ }^{3}$ \\ ${ }^{1}$ Assistant Professor, American International University-Bangladesh (AIUB) \\ ${ }^{2}$ Assistant Professor, American International University-Bangladesh (AIUB) \\ ${ }^{3}$ BBA Student, American International University-Bangladesh (AIUB)
}

\begin{abstract}
Over Tk. 1,000 crore annual turnover in 2016, 60 Million internet users and 50,000 actively engaged people of Bangladesh in the e-commerce industry, eventually acquiring global attention. Studies indicated that for technological advancement and customers' interests, "online shopping" is getting good acceptance. When electronics and fashion industry receives preference to online marketers, a viable segment "Online Grocery Shopping" is lagging. Existing online grocery sellers still cannot cover its service in specified areas. To analyze the market potential, this study tried to identify consumers' awareness and acceptability towards online grocery shopping. This research examined consumers' knowledge and acceptance; current purchasing patterns; existing facilities provided and expected benefits of the consumers, and prospects and limitations of online grocery in specified areas. 200 respondents were interviewed with a structured questionnaire with the reliability of 0.762. Factors affecting purchasing grocery items and the existing limitations of online grocery platform were identified and analyzed. Responses were analyzed with SPSS 17.0 using Frequency, Mean and Standard Deviation. It has been identified that respondents are aware and interested towards online grocery shopping, where convenience is the priority when they shop online. Reliability and trustworthiness are the most expected factors from the online shopping companies, adaptation to new technology is positive and indicates that "online grocery shopping" have prospects and sustainability. It is perceived that; the infrastructural facilities and sociocultural environment require more advancement and support in favor of such trade. This study will assist the existing and prospective online shopping companies to understand consumers' buying behavior in depth.
\end{abstract}

Key Words: Online Grocery Shopping, Awareness and Acceptability, Convenience, Reliability, Trustworthiness

\section{Introduction}

Retailing has always offered a variety of shopping options to the consumers [1]. In this modern era, retailing via internet is one of the most rapidly growing means of trade [2][3][4], the ongoing development of communication technologies, particularly internet, has increased both the retailers' potential to reach consumers all over the world and the consumers' access to these retailers [5]. Due to the rapid growth of ICT, online shopping has been a cause of excitement and attention for consumers to explore new dimensions of product options [6]. The pattern of the retail stores is changing as the growing number of retailers shift their attention to the emerging economies [7]. It is expected that Amazon's recent entry into South-East Asian retail markets will change the business dimension and traditional retailers will be the ultimate suffers [8]. To keep the pace with regional economies, the online based business sectors in Bangladesh are also growing at an extensive rate. According to the Bangladesh Association of Software and Information Services (BASIS), more than 2,000 
online trading companies have entered Bangladesh in the last three years [9]. With the e-commerce industry making its mark in Dhaka, the Capital City of Bangladesh, there are ample opportunities for online groceries [10]. This study aims to investigate and analyze the level of consumers' awareness and acceptability of online grocery shopping among the consumers of the Dhaka Metropolitan City. It has focused on the existing features that motivated them to buy grocery items from the online sources in comparison with the traditional markets. However, challenges, scopes and limiting factors of online grocery shopping spheres are to be highlighted in this study and new dimensions will be explored considering the growth potentiality of the online based grocery shopping by the consumers.

\subsection{Objectives of the Study:}

- To study the consumers' awareness, knowledge, and acceptability towards online grocery shopping in Dhaka Metropolitan City area.

- To analyze consumers' existing patterns of purchasing grocery items from the traditional and online market platforms.

- To find out the differences between the current market offerings and consumers' expectations from the online marketers.

- To evaluate the market prospects and limitations of online shopping market platforms in the Dhaka Metropolitan City area.

\section{Review of Related Literature}

The online platform is becoming a significant part of our daily life activities. This characteristic of global marketing created a new window for retailers to reach existing and potential customers more efficiently by replacing traditional retail trades to online retail stores [2]. In 2016, the worldwide retail e-commerce sales have reached approximately $\$ 1.915$ Trillion and in 2020 the sales will touch at $\$ 4$ Trillion [11]. As the competition in e-commerce industry is getting influential gradually, it is important for online retailers to know the consumer's behavior and their expectations towards online shopping and eventually retain them towards the new dimension of the online trade [12]. From previous studies, it has been observed that consumers prefer online shopping because of convenience, time-saving, best prices, availability of product options and better customer services [13]. Online business in Bangladesh is a potential and viable sector, and to facilitate this sector some measures must be taken such as educating customers towards technology adoption and increasing their shopping intentions from the online platforms. The government should facilitate the infrastructural benefits and the online transactions should be widened and secured [14]. However, to raise the acceptance level among consumers building trust is necessary, on-time delivery should be maintained, products return policy has to be ensured [15]. For increasing satisfaction level of the online customers, different measures like website design, product varieties, and service reliability are considered as the key elements [16].

\section{Methodology of the Study}

The research is applied in nature because of its narrow focus on the problem, area of investigation and geographic concentration. As the researchers tried to figure out the certain behavioral pattern of consumers through the survey towards the specific problems, it is falling into the category of "Descriptive" form of research. To facilitate the research objectives, secondary data were collected from journals, research papers, reliable news articles, and governmental online portals. Primary data were collected using simple random sampling, from face to face interviews of 200 potential consumers of the selected area. 'Nominal Scale' questions were asked to understand the demographic profile of the customers, and 'Interval Scale' questions were asked to know about the income level, expenses, buying patterns, etc. Five points 'Likert Scale' questions were asked to understand the level of consumers' interests, acceptability, and sustainability towards online 
grocery shopping. Lastly, 'Category Scale' questions were asked to investigate the research objectives. Collected responses were analyzed using SPSS 17.0 using frequency, mean and standard deviation. Suggestions are given based on statistical findings from the responses and related literature of the study from the potential customers.

\section{Analysis and Findings:}

It is essential to know how customers desire to buy products from the online platforms. The responses showed that $28.9 \%$ of consumers want to buy "Occasionally", $27.5 \%$ "weekly" and only 6\% on "Daily" basis. Usually, they are habituated to purchase grocery items after a certain time interval from the traditional markets, for online they have shown the similar attitude. The average monthly expenses for grocery is Bangladeshi Taka (BDT) 6,001 to 12,000 , then in each transaction consumers want to spend around BDT 1,001 to 1,500 on online purchase. Almost half of the respondents $(47.3 \%)$ were private service holders and income level of these respondents varied between BDT 35,000 to 57,500 per month approximately.

TABLE I: Consumers’ Awareness and Acceptability, Business Prospects and Sustainability

\begin{tabular}{|l|c|c|}
\hline \multicolumn{1}{|c|}{ Items } & Mean & Standard Deviation (SD) \\
\hline Level of knowledge or awareness about “online grocery shopping” & 3.72 & 1.00220 \\
\hline Interest towards “online grocery shopping” & 3.61 & 0.866982 \\
\hline Level of willingness to adopt new technology regarding online grocery shopping & 4.41 & 0.65884 \\
\hline Satisfaction with existing infrastructural facilities related to online grocery shopping & 3.33 & 0.88033 \\
\hline Observation about socio-cultural development towards online grocery shopping & 3.49 & 0.82057 \\
\hline Prospects and sustainability of online grocery shopping in Dhaka Metropolitan city area & 4.03 & 0.814 \\
\hline
\end{tabular}

To know the consumers' awareness level regarding their purchase intention to buy grocery items from the online platforms is an important factor to be considered. Mean value of 3.72 indicated that the respondents are close to "Aware" and therefore marketers' need to take some initiatives to create a substantial level of awareness. The SD value showed a positive result, therefore indicated that the responses were mostly homogenous in nature regarding the level of awareness and less variation in their responses.

$>$ To know the level of interest among the consumers towards online grocery shopping, a mean value of 3.61 is considered as a "Moderate" level and indicated that the respondents were interested towards online grocery shopping in the urban areas. In terms of the SD calculation, the result also showed that majority of the respondents has given similar responses towards their interest level.

$>$ To understand the technological adaptability among the consumers, a mean value of 4.41 specified that the respondents were more than "Adaptable" towards technological advancements. The SD value represented the respondents' intention towards adaptability which was also similar in nature. To support this statement, a study has shown that around 82, 00,000 smartphones have been imported in Bangladesh in the year 2016 [17]. This signifies that there is a huge market for smartphones in Bangladesh and people are willing to adopt new technologies for communication and trade.

$>$ To foster online based trade in any country, the existing infrastructural facilities are considered as a vital factor. When the respondents were asked about the related infrastructural facilities available in the urban areas of Bangladesh, a mean value of 3.33, which can be referred as "Moderately Satisfied" so there are still some scopes for further improvement. The SD value showed a homogeneous response and indicated that the existing infrastructural facilities are not quite satisfactory due to inadequate internet services provided to the users.

$>$ To understand the viability of any business to operate in a specific country, sociocultural values are very essential to consider. A Mean value of 3.49 indicated that the respondents have given a "Moderate" response and the SD value showed a pattern that the maximum respondents have agreed that the sociocultural views towards online shopping are optimistic. The findings referred that the existing social beliefs are less favorable 
towards online grocery shopping in the urban areas and such beliefs can influence independently or can influence jointly together with the variables of economic condition and personal factors [18].

$>$ For understanding the dynamics of customer preferences, it is essential to know the sustainability of online based trades in the business sector. A mean value of 4.03 emphasized the sustainability of online grocery shopping is "Potential" in the urban markets of Bangladesh. The SD value showed the consistency among the respondents and emphasized on the growth potentiality in the market.

$>$ In reference to the question about their preference in online shopping, the respondents were asked to scale their preferences from Very Important to Least Important. A mean value of 3.00 indicated "Moderately Important" to the variables given. Among the variables mentioned, "Convenience" factor has got the highest preference and the SD value showed a homogeneous response to the given factor. The respondents have given their replies that through the application of online platforms in trade, it is saving time and energy of the consumers and they can purchase their desired products at ease.

$>$ When the respondents were asked about their preferences in selecting their product options while purchasing from the online platforms, most of the mean values in the category showed positive results indicating "Moderately Preferred". Among the given categories, "Biscuits and Snacks", got the highest preference and simultaneously the SD value showed the lowest preference for the other variables. The SD values of the other variables showed a variation in their choice of products where "Meat and Fish" and "Fruits and Vegetables" have achieved the least responses among the given options. These categories have the characteristics of being the most perishable products among the products given. A study indicated that online buyers for grocery items have not given their best response in favor of perishable items and this can be a major reason for worldwide limitations of online grocery sales [19].

$>$ In response to the question asked about the preferred payment method, the mean value of 4.63 highlighted that the "Cash on Delivery" method is the most "Preferred" means of payment. From the local market perspective, consumers have a strong preference towards "Cash on Delivery" and have shown uniformity among the respondents. A study has shown that $83 \%$ of consumers in India, trading on the online platforms, prefer to use "Cash on Delivery" as a popular means of the transaction [20]. However, the findings showed "Mobile Banking" and "Debit Card" payment methods are still preferred but the use of "Credit Card" method has obtained the least preferred means of payment method.

$>$ When the respondents were asked about their preference in the communication medium, the mean value of 4.64 showed that the "Social Media and Digital Platforms" and "Influence of Reference Groups" have achieved the highest preferences. The SD values have also indicated homogeneity in their responses. Online marketers prefer the social media as a brand building tool which can be utilized as a direct product marketing platform [21]. The reference groups also influence the consumer's buying behavior to a wider dimension. However, "Email" and "SMS marketing" are also accepted by the consumers whereas "Sales Representative" showed the least preferred option as a medium of communication, especially for online grocery trade.

$>$ In response to the question about the existing limitations which are needed to be solved, respondents have marked maximum on "Visualized and Real Product Differences". The mean value of 4.74 indicated close to "Very Important" and the SD showed the similarity of opinions among most of the respondents. "Physically Untouchable" and "Bargaining Impossibility" also received accepted values in mean and SD scores.

$>$ When the respondents were asked about their expected benefits from the online marketers, the maximum responses came towards "Reliability and Trustworthiness". The mean value of 4.69, indicated close to "Very Important", concurrently the SD value is the lowest among the variables. Variables such as increased distribution network, comparatively low price, consumer awareness building programs, CRM, and publicity, indicated towards "Important" for these variables which can be incorporated by the marketers. 


\section{Suggestions for Further Implications}

Some of the suggested views for further implications by the researchers are mentioned below:

$\square$ Since the awareness level still could not reach the optimum level in Dhaka Metropolitan City, therefore for promotional purposes, social media and digital platforms should be given the first priority by the marketers. Although the initial findings of this study indicated that most of the consumers got familiarity about online grocery from the mass media but for upcoming marketing communications, they prefer the social media platforms. Moreover, marketers should utilize their loyal customers for motivating their family members, friends, colleagues and peer groups to buy grocery items from the online platforms. For making it mutually beneficial, marketers can offer special benefits such as membership cards and complimentary products for the new customers, and reward points for the existing customers for conveying new customers to the business.

$\square$ Marketers need to focus on improving services to give comfortable online shopping experiences. In such case, a good User Experience can be given while designing user-friendly web portals and mobile applications (Apps). Delivery time should be made more competent so that customers can buy from the online platforms spending comparatively lesser time than from the traditional markets or from the super shops.

$\square$ Building trust with the customers and giving reliable services can be one of the important tasks for online grocery marketers which may include, giving the assurance of quality products, promising competitive price of the traded products, ensuring effective post-sales service, etc. Marketers need to work on brand engagement activities, where few exclusive physical outlets can be established in prominent areas of the Dhaka City. The outlet's sales representatives can demonstrate to the visitors about the fresh product offerings and especially mention that the same products can also be ordered 24x7x365 from the specific online portals and mobile apps. Prominent celebrities can be endorsed by the online marketer to promote the authenticity and freshness of their products.

$\square$ Although "Cash on Delivery" is widely accepted by the consumers but it possesses the less sustainability in terms of online shopping. The reason behind this is, the ultimate meaning of online shopping is to buy with electronic money and generating profit from the alliance with different financial services company. Payment over mobile banking such as the application of mobile wallets which will be beneficial for the customers, marketers and the financial companies due to prompt payment and growth in financial transactions.

$\square$ The IT infrastructure and support facilities can be enhanced. For instance, easy access to the internet should be developed by the government and non-government organizations to facilitate e-commerce, which will leverage the online grocery shopping sphere. The cost of the internet tariffs from telecommunication companies can be reduced to increase the internet usage among the users and upcoming $4 \mathrm{G}$ internet connectivity should be stable and reliable enough to carry out faster online transactions.

$\square$ There are some prevailing social beliefs about the online grocery shopping in the urban areas of Bangladesh, for which marketers need to strongly focus on those concerns. Many of the residing non-users of online shopping believe that online shopping resembles high price of the products available. Some consumers perceive that it is better to purchase perishable items such as fishes, meat, fruits, and vegetables from the traditional markets as they can physically verify its texture and freshness. Therefore, online marketers need to highlight their credible sourcing of the grocery items for minimizing the misconception among target groups. The visualized picture of these grocery items in the web pages must have maximum similarity with the delivered products to avoid any kind of post-purchase dissatisfaction among the customers.

$\square$ On increasing the buying frequency of the consumers, two types of strategies can be applied by the marketers, the short term strategy is where the marketers can give sales promotional offers on the weekly and occasional basis as consumers usually purchase grocery items between this interval. For the long term strategy, marketers should establish a perception in the mind of the consumers that, now by the click of a computer's mouse, it is convenience to purchase on a regular basis despite being busy with their daily activities. By practicing such strategies, marketers will be able to sell frequently and in sufficient amount and buyers can avail the freshness of the products purchased from the online platforms. 


\section{Conclusion}

Marketing in Metropolitan areas has always been a challenging task because of the large deviation in demographical characteristics such as in Dhaka, the Capital City of Bangladesh, where people influxes from the 64 districts of the country. This study determined the feasible clarifications for the online grocery marketers by stating that the consumers' interests are sufficient to target, as they are aware and ready to adopt the necessary technological changes to purchase grocery items from the online platforms. Furthermore, this study has also discussed the probable solutions for the internal limitations of online shopping marketers and external infrastructural constraints. From the overall study, it can be inferred that online grocery shopping has got prospects and sustainability in Dhaka Metropolitan City area. Any future investment or entry of online grocery marketers will be accepted by the consumers of the specified area. However, for further studies, researchers can give concentration on the marketer's perspective for identifying a sustainable online grocery supply chain model.

\section{References}

[1] Wilson-Jeanslme, M. and Reynold, J. 2006. "Understanding shoppers' expectations of online grocery retailing", International Journal of Retailing and Distribution Management, Vol. 34, Issue: 7, pp.529-540. https://doi.org/10.1108/09590550610673608

[2] Limayem, M., Khalifa, M., and Frini, A. 2000. "What makes the consumer buy from the internet? A longitudinal of online shopping", IEEE Transactions on Systems, Man, and Cybernetics, Volume: 30, Issue: 4, Jul 2000.

[3] Levy, M., Weitz, B., and Grewal, D. 2014. "Retailing Management", $9^{\text {th }}$ Edition, McGraw Hill Education.

[4] Shim, S., Easstlick, M.A., Lotz, S. and Worrington, P. 2001. "An online purchase intention model: The role of intention to search", Journal of Retailing 77, pp. 397-416.

https://doi.org/10.1016/S0022-4359(01)00051-3

[5] Ilagan, S.V. 2009. "Exploring the impact of culture on the formation of consumer trust in internet shopping", Department of Communication and Journalism and The Graduate School of the University of Wyoming.

[6] Aylott, R., and Mitchell, W.V. 1998. "An exploratory study of grocery shopping stressors", International Journal of Retail \& Distribution Management, Vol. 26, Issue: 9, pp. 362-373.

https://doi.org/10.1108/09590559810237908

[7] Goldman, A. 2001. "The transfer of retail formats into developing economies: The Example of China", Journal of Retailing, Vol. 77, Issue: 2, Summer 2001, p.p. 221-242. https://doi.org/10.1016/S0022-4359(01)00044-6

[8] Balea, J. July 2017. "Winners and losers in Amazon entry into Southeast Asia", Tech Asia [Online], Available: https://www.techinasia.com/winners-losers-amazon-entry-southeast-asia

[9] “Kaymu: How Bangladesh's Number One Online Marketplace Changing the E-commerce Trend”. January 2015. Future Startup [Online], Available: http://futurestartup.com/2015/01/15/kaymu-bangladesh-e-commerce-trend/

[10] Haque, M. April 2015. "Bangladesh: Online Grocery shopping on the rise", SD Asia [Online], Available: https://sdasia.co/2015/04/01/bangladesh-online-grocery-shopping-on-the-rise/

[11] "Worldwide Retail Commerce E-commerce Sales Will Reach \$1.915 Trillion This Year”, August 2016, eMarketer [Online], Available: https://www.emarketer.com/Article/Worldwide-Retail-Ecommerce-Sales-Will-Reach-1915Trillion-This-Year/1014369

[12] Zhou, L., Dai, L., and Zhang, D. 2007. "Online Shopping Acceptance Model- A Critical Survey of Consumer Factors in Online Shopping”, Journal of Electronic Commerce Research, Vol. 8, No. 1, 2007, p.p. 41-62.

[13] Howlader, M.H.R., Moinuddin, M.G., and Islam, M.M. 2012. "Developing Online Shopping Intention among People: Bangladesh Perspective”, IISTE, Vol 2, No.9, 2012, p.p. 69-76. 
[14] Suhan, J. 2015. “Acceptance of Online Shopping in Bangladesh: Consumer's Perspective”, IOSR-Journal of Business and Management (IOSR-JBM), Vol. 17, Issue 1.Ver. II (Jan. 2015), p.p. 14-24.

[15] Pachauri, M. 2002. "Researching Online Consumer Behavior: Current Position and Future Perspectives", Journal of Customer Behavior, Vol. 1, No. 2, June 01, 2002, pp. 269-300.

https://doi.org/10.1362/14753920260595246

[16] Alam, S.S., and Yasin, N.M. 2010. "An Investigation into the Antecedents of Customer Satisfaction of Online Shopping”, Journal of Marketing Development and Competitiveness, Vol. 5, Issue 1, 2010, p.p. 71-78.

[17] "Smartphone Imports Soar 46pc", February 2017, The Daily Star [Online], Available: http://www.thedailystar.net/business/smartphone-imports-soar-46pc-1360750

[18] Lawan, L.A., and Zanna, R. 2013. "Evaluation of Socio-Cultural Factors Influencing Consumer Buying Behavior in Borno State, Nigeria”, International Journal of Basic and Applied Sciences, Vol.1, No. 3, p.p. 519-529. https://doi.org/10.17142/ijbas-2012.1.3.7

[19] Kestenbaum, R. January 2017. "Why online grocers are so unsuccessful and what Amazon is doing about it", Forbes [Online], Available: https://www.forbes.com/sites/richardkestenbaum/2017/01/16/why-online-grocers-are-sounsuccessful-and-what-amazon-is-doing-about-it/

[20] "Cash on Delivery Remains the Preferred Method of Payment in India". January 2016. Business Insider [Online], Available: http://www.businessinsider.com/cash-on-delivery-remains-the-preferred-method-of-payment-in-india2016-6

[21] Alkerton B. July 2010. “Using Social Media to Promote Your Online Store”, Spotify Blog [Online], Available: https://www.shopify.com/blog/3033032-using-social-media-to-promote-your-online-store 